\title{
Copy Number Analysis Tool
}

National Cancer Institute

\section{Source}

National Cancer Institute. Copy Number Analysis Tool. NCI Thesaurus. Code C81017.

A software application that performs copy number and loss of heterozygosity ( $\mathrm{LOH}$ ) analysis on data from Affymetrix mapping genotyping microarrays. 